027 CHANGING INFLUENCES ON CHILDHOOD OBESITY: A STUDY OF TWO GENERATIONS OF THE 1958 BRITISH BIRTH COHORT

L Li, A Pinot de Moira, C Power. MRC Centre of Epidemiology for Child Health, UCL Institute of Child Health, London, UK

doi:10.1136/jech.2009.096719a

Objective: To investigate whether risk factors for childhood obesity have changed over time, either in their strength of influence or prevalence.

Design: Prospective cohort study.

Setting: Great Britain.

Participants: The 1958 birth cohort and a sub-sample of their offspring. Height and weight measures, and information on prenatal and early life factors were collected on cohort members aged 7 years in $1965(\mathrm{n}=8552)$ and on offspring aged 4-9 years in 1991 ( $\mathrm{n}=1889$ ).

Main Outcome Measure: Body mass index (BMI) standard deviation scores (SDS) and overweight/obesity, derived using international standards.

Results: Prevalence of childhood overweight/obesity increased by more than $50 \%$ between generations. Higher parental BMI was associated with increased childhood BMI; for example, cohort members and offspring of obese mothers had more than double the risk of overweight/obesity, with adjusted odds ratios of 2.29 (95\% CI 1.76 to 2.97 ) and 3.20 (1.85 to 5.54) respectively. Maternal smoking and smaller family size were associated with higher childhood BMI in both generations. There was evidence suggesting that social gap in childhood obesity was widening: indicators of lower socio-economic position showed either no association or a protective effect in cohort members, but tended to be associated with increased BMI in offspring. Full-time maternal employment was associated with greater BMI among offspring, for example by $0.42 \mathrm{~kg} / \mathrm{m}^{2}$ in boys, an effect that had strengthened between generations $(p<0.05)$. Several risk factors had altered in prevalence: parental obesity and maternal employment had increased; socioeconomic factors had improved; family size and maternal smoking had reduced.

Conclusions: Risk factors for childhood obesity had altered across two generations, either in terms of their strength of association, their prevalence or both. Parental obesity, maternal employment and socio-economic factors may be playing an increasingly important role in the childhood obesity epidemic.

\section{DOES CHILDCARE INFLUENCE THE RISK OF OVERWEIGHT AND OBESITY IN THE EARLY YEARS? FINDINGS FROM THE UK MILLENNIUM COHORT STUDY}

${ }^{1} \mathrm{~A}$ Pearce, ${ }^{\mathrm{L}} \mathrm{LLi},{ }^{2} \mathrm{~J}$ Abbas, ${ }^{2} \mathrm{~B}$ Ferguson, ${ }^{3} \mathrm{H}$ Graham, ${ }^{1} \mathrm{C}$ Law, the Millennium Cohort Study Child Health Group. ${ }^{1}$ MRC Centre of Epidemiology for Child Public Health, London, UK; ${ }^{2}$ York and Humberside Public Health Observatory, York, UK; ${ }^{3}$ Department of Health Sciences, University of York, York, UK

\section{doi:10.1136/jech.2009.096719b}

Objectives: To explore whether childcare type (parent, informal, formal) is associated with overweight in children, and whether this differs by socio-economic background.

Design: Nationally representative prospective cohort study.

Setting: Children born in the UK in 2000-2002.

Participants: 12354 children with information on childcare between 9 months and 3 years, and height and weight data at age 3 .

Main Outcome Measure: Overweight (including obesity) at age 3 defined by the International Obesity Task Force cut-offs for body mass index.

Results: Children who were cared for in informal childcare were more likely to be overweight than those cared for by a parent (risk ratio ( $R R$ ) $1.14,95 \%$ CI 1.04 to 1.25 ), particularly if they were in full-time child care (RR 1.36, 1.16 to 1.59). The risk of overweight in children who were cared for in formal childcare did not differ from those cared for by a parent (1.05, 0.95 to 1.15$)$. These associations remained after controlling for confounders. When stratifying by socio-economic background the increased risk of overweight in informal childcare (compared to parental care) was limited to children from more advantaged groups. Children were at increased risk of overweight if they were cared for in informal childcare and their mother was from a managerial or professional background (RR $1.24,1.04$ to 1.47 ), had a degree (RR 1.48, 1.19 to 1.84), or lived in a couple household (RR 1.16, 1.05 to 1.28), whereas there was no increased risk seen for informal childcare if the mother was from a routine or manual background, had no educational qualifications or was a lone parent. Further exploration revealed that the increased risk associated with informal childcare was experienced by children who were cared for by grandparents $(1.15,1.04$ to 1.27$)$ and not in other types of informal care. However, after taking into account hours per week spent in childcare, an increased risk was seen for full-time care by grandparents (RR 1.34, 1.12 to 1.56) (and not parttime care), and also for other full-time informal childcare (RR 1.41, 1.09 to 1.82 )

Conclusions: Evidence from the US indicates that informal childcare is associated with a greater risk of childhood overweight. Our findings are consistent with this, although only for children from more advantaged groups. Whilst further research is required, these results suggest that the UK government's drive to support parents into paid employment should be accompanied by health related information and support for informal as well as formal carers such as nurseries. The recent announcement to provide grandparents with National Insurance credits for caring for grandchildren provides a potential opportunity for health promotion.

\section{Trends in CVD}

\section{STROKE MORTALITY TRENDS FOR ENGLAND: MODELLING THE PAST TO PREDICT THE FUTURE}

CJ Sutton, J Marsden, CL Watkins, MJ Leathley, P Dey. University of Central Lancashire, Preston, UK

\section{doi:10.1136/jech.2009.096719c}

Background: In the UK, overall stroke mortality has declined. It is important to planners of health service provision to understand how trends in stroke mortality are changing and, given changes in population structure, how trends in stroke mortality will affect the future burden on the nation. We therefore aimed to investigate recent stroke mortality trends amongst those aged over 40 years in England and to make predictions of stroke mortality to 2015.

Methods: We obtained annual aggregated stroke death (ICD-10 I60 to I69) and population data for England from the Office of National Statistics for the years 1979-2005 and used these data to investigate time trends in gender-specific mortality rates for adults aged over 40 years. We applied log-linear modelling to identify effects attributable to age, linear "drift" over time, non-linear period and birth-cohort effects; discontinuities in "drift" were also considered. Different forms of model were compared for relative goodness-of-fit using Akaike's Information Criterion and residual analysis was used to identify specific aspects of lack of fit of individual models. Predictions to 2015 were made for the best-fitting models.

Results: Stroke mortality predictions were not particularly sensitive to many of the aspects of the underlying choice of model, with, for example, the more complex models including different age and period effects for males and females providing similar predictions to simpler models. However, predictions were highly sensitive to varying assumptions about potential discontinuities in 